\title{
Alterations of cAMP/cGMP Signaling Pathways in Lupus Nephritis Issaka Yougbare*
}

\section{Article Info}

Article Notes

Received: June 14, 2021

Accepted: August 06, 2021

\section{${ }^{*}$ Correspondence:}

*Dr. Issaka Yougbare; Email: Issaka.Yougbare@sanofi.com.

${ }^{\circledR} 2021$ Yougbare I. This article is distributed under the terms of the Creative Commons Attribution 4.0 International License.

\section{Keywords}

CAMP

CGMP

PDE1

PDE4

Chronic inflammation

Cell proliferation

Kidney remoldeling

Lupus nephritis

\begin{abstract}
Systemic lupus erythematosus (SLE) is an autoimmune disease with a broad spectrum of clinical manifestations, but its pathogenesis remains fairly understood. Cyclic nucleotide signaling pathways in immune cells and kidney are emerging as cellular mechanisms governing SLE disease progression. Upregulations of cGMP/cAMP metabolism lead to lupus nephritis and abnormal kidney remodeling/hypertrophy. PDE4 family remains the major CAMP hydrolyzing enzyme as PDE1 is responsible for CGMP breakdown in kidney. SLE disease progression to lupus nephritis is correlated with increase PDE1 and PDE4 activities resulting in lower cyclic nucleotide levels in kidney. Administration of Nimodipine, a PDE1 inhibitor prevents the lymphoproliferative phenotype and exert anti-proliferative effects on mesangial cells while PDE4 inhibitor NCS 613 prevents inflammatory cytokines release, immune complex deposition, and nephritis in MRL/Ipr lupus prone mice. In this review, we highlight recent findings of alterations of cyclic nucleotide signaling pathways in lupus nephritis. Given the role of cAMP/cGMP signaling in kidney function, dual inhibition of PDE1 and PDE4 may represent a promising therapeutic approach to tackle lupus nephritis.
\end{abstract}

\section{Introduction}

Systemic lupus erythematosus (SLE) is an autoimmune disease with a broad spectrum of clinical manifestations. Secretion of variety proinflammatory cytokines and autoantibodies is the hallmark of SLE. Loss of tolerance to endogenous antigens initiates generations of antibodies against nuclear compartment including spliceosomes, DNA, and phospholipids ${ }^{1}$. This polymorphic and multigenic autoimmune disease predominantly affects women in their premenopausal age. Although several organs can be affected, lupus nephritis (LN) remains the major cause of morbidity and mortality in patients with SLE and affects up to $60 \%$ of patients ${ }^{2}$. However, the immunopathogenesis of LN is elusive since key mechanisms explaining kidney cell injury and organ failure remains largely unclear. It has been reported that podocyte injury after deposition of immune complexes leads to irreversible glomerular damage ${ }^{3}$. There is a positive correlation between anti-dsDNA antibody and IL-6, IL-10, and TNF- $\alpha$ levels which can affect the prognosis of SLE ${ }^{4}$. The deposition of immune complexes in different areas of the glomerulus activates components of the immune system as well mesangial cell proliferation $^{5}$. Recent studies focusing on immune cell landscape of lupus nephritis have revealed subsets of leukocytes active in this disease. Macrophages, T cells, natural killer cells, and B cells demonstrated both pro-inflammatory responses and inflammationresolving responses ${ }^{6}$. Cyclic nucleotides (cyclic adenosine-3',5'monophosphate (cAMP) and cyclic guanosine-3',5'-monophosphate (cGMP)) have been long recognized as critical mediators of many 
renal functions, including solute transport, regulation of vascular tone, proliferation of parenchymal cells, and inflammation ${ }^{7-9}$.

Intracellular calcium handling is unbalanced in $\mathrm{T}$ lymphocytes from lupus patients which lead to overactivity of $\mathrm{T}$ cells. In addition, it has been reported that a low intracellular cAMP level is associated with impaired cAMPdependent phosphorylation of endogenous substrates in $\mathrm{T}$ lymphocytes from patients with active disease. Therefore, SLE is characterized by exaggerated T-cell activity and abnormal T-cell signaling. The mitogen-activated protein kinase (MAPK) pathway is involved in the maintenance of T-cell tolerance ${ }^{10}$. We have previously demonstrated that inhibition of cAMP degrading enzyme can prevent proinflammatory signaling including MAPK and nuclear factor $\kappa \mathrm{B}(\mathrm{NF} \kappa \mathrm{B})$ in peripheral leucocytes from of patients with $\mathrm{SLE}^{11}$. Other clinical future of lupus nephritis is mesangial cell proliferation and podocyte loss mediated by immune complex deposition ${ }^{12,}{ }^{13}$. Mesangial cell proliferation is increased in presence of low intracellular cGMP, therefore elevated glomerular cGMP levels has been shown to inhibit of mesangial proliferation, glomerular matrix accumulation, and proteinuria ${ }^{8,14}$. This review will discuss the role of cyclic nucleotide signaling pathways in lupus nephritis complications. This manuscript will present current understanding of LN from lessons learned from animal models and interesting therapeutic approaches for the management of SLE.

\section{Cyclic Nucleotides Signaling in Kidney}

Cyclic nucleotide signal transduction pathways are an emerging research field in kidney disease. Downstream receptor activation, cyclic nucleotides are second messengers that govern many physiological processes and pathology including inflammation and cell proliferation ${ }^{15}$. cAMP and cGMP are synthesized by adenylyl cyclases and guanylyl cyclases respectively. cAMP and cGMP participate in kidney physiological responses and have been shown to be renoprotective ${ }^{16}$. Cyclic nucleotide intracellular signaling pathways are regulated by cyclic nucleotide phosphodiesterase families (PDE1 to PDE11) that hydrolyze cAMP and cGMP as a feedback mechanism to return to basal levels. The balance of intracellular cyclic nucleotide levels is mainly dependent on the anabolic activity of adenylyl/guanylyl cyclase and the catabolic activity of PDEs. In fact, the hydrolytic activity of PDEs against cyclic nucleotides is greater than that of synthesis. Activation of PDEs abolishes the cellular effects of cAMP and cGMP signaling, while inhibition of PDEs increases cAMP and/or cGMP signaling. The superfamily of PDEs encompass more than 100 identified proteins which participate in their functional diversity. The multiplicity of PDEs and their specific intracellular localization in the vicinity of various protein effectors contribute of the compartmentalized regulation of cAMP and $\mathrm{cGMP}^{17,18}$. By regulating cyclic nucleotide levels, PDEs contribute to their compartmentalization and govern many biological processes including inflammation, cell adhesion, proliferation and migration ${ }^{19}$.

In disease conditions such as glomerulosclerosis and nephritis, increased cAMP or cGMP have been shown to suppress these renal diseases. Brain natriuretic peptide (BNP) induces cGMP signaling by stimulating guanylyl cyclase A. Through activation of cGMP-dependent kinases and an increase intracellular calcium, BNP elicits vasodilating, natriuretic effects, inhibition fibroblast proliferation, and anti-proliferative effects on mesangial cells. Elevated the renal content of cGMP can result in an improved renal plasma flow, glomerular filtration rate, and sodium excretion. In cirrhotic animals, it has been previously shown that PDE5 inhibitor ameliorated the sodium retention and the resistance to the natriuretic effects of ANP ${ }^{14,20}$. The cAMP pathway exerts anti-fibrotic actions which include inhibition of epithelial mesenchymal transition (EMT), blockade of fibroblast proliferation, and activation of the death of fibroblasts ${ }^{21}$. TGF $\beta$ levels play a dual role in the pathogenesis of lupus nephritis with immunoregulatory and proinflammatory properties dependent on different microenvironments ${ }^{22}$. The antifibrotic effects of cAMP are mediated by PKA through cAMP responsible element binding (CREB) phosphorylation which blocks TGF $\beta$ mediated gene transcription. It is well accepted that activation exchange protein directly activated by cAMP (Epac) also blocks TGF $\beta$ mediated smad dependent gene transcription thereby the EMT.

\section{PDE4, the Major cAMP Degrading Enzyme in Lupus Nephritis}

The clinical complications of lupus include nephritis, neurological disorders, and dermatitis. Lupus nephritis has been described as the most serious complication of SLE and strongest predictor of poor disease outcomes. Lupus prone mice (MRL/lpr) strain which bears an autosomal recessive mutation in the gene encoding Fas, is widely used as animal model for autoimmune disease ${ }^{23}$. The $\mathrm{MRL}^{+/+}$background is responsible for the development of autoimmune kidney disease, and the lymphoproliferation (lpr)/Fas mutation converts a mild nephritis into a much severe disease, with a $50 \%$ mortality rate at 24 weeks of age ${ }^{24}$. Although this molecular defect in Fas gene does not lead to SLE in human, this mouse model recapitulates many biological, immunological and clinical features of the human disease.

Among PDEs, the members of the PDE4 family (PDE4A, PDE4B, PDE4C and PDE4D) specifically hydrolyze cAMP and are mainly present in inflammatory cells ${ }^{11,25}$. PDE4 family comprises of 4 genes with various alternative mRNA splices encoding long and short PDE4 isozymes, resulting 
in 35 different proteins ${ }^{15,26}$. Peripheral leukocytes from mice deficient in PDE4B loss approximately $90 \%$ of TNF $\alpha$ production, a major proinflammatory cytokine is SLE pathogenesis. Renal injury in LN occurs through either immune complex deposition, autoantibodies binding to glomerular antigens, or in situ immune complex formation within glomeruli ${ }^{2}$. Early study in MRL/lpr mice established the involvement of PDE4 family in lupus disease progression. Kidney cAMP is degraded by PDE2 and PDE3, but predominantly by PDE4 family which contributes to $66 \%$ of cAMP-PDE activities. In the kidney of young healthy mice, all 4 subtypes of PDE4 are expressed (PDE4A, PDE4B, PDE4C, and $P D E 4 D)$. cAMP regulation mediated by the differential expressions of PDE4 contribute to kidney inflammation. MRL/lpr mice spontaneously develop systemic inflammation and nephritis when transitioning from young (week 8) to adult life (week 15). It should be noticed that disease progression is correlated with significant increase in cAMP catabolism by PDE4 without change in PDE2 and PDE3 activities. The only PDE4C isoform expressed in the kidney is absent in lupus prone mice, while PDE4B isoform is upregulated. Interestingly, NCS 613 a PDE4C selective inhibitor can reduce TNF $\alpha$ secretion, prevent proteinuria, and improve survival in lupus prone mice. NCS 613 restores renal PDE4C expression, a membrane associated PDE4. Other PDE4 inhibitors including pentoxifylline and denbufylline do not improve animal survival but reduce TNF $\alpha$ secretion. NCS 613 is a new potent PDE4 inhibitor which exerts anti-inflammatory properties (Figure 1). Oral administration of NCS 613 reduces neutrophil recruitment in lipopolysaccharide (LPS)-treated mouse bronchi as and abolishes hyperresponsiveness of cultured human bronchi ${ }^{27-29}$. Recently, we reported that NCS 613 suppresses systemic inflammation and immunes complexes deposition in kidney of lupus prone mice ${ }^{30}$. Apremilast is an orally available small molecule PDE4 inhibitor approved by the USA FDA for the treatment of active psoriatic arthritis and moderate-to-severe psoriasis. Apremilast has been shown to block production of IL-12, IL-23, TNF-a, INF- $\gamma$ by leukocytes with subsequent suppression of Th1 and Th17-mediated immune responses ${ }^{31}$. NCS 613 targets immunocompetent cells including $\mathrm{T}$ cells, $\mathrm{B}$ cells, and phagocytes to achieve its therapeutic effects in controlling systemic inflammation. As an impact, systemic secretion of TNF $\alpha$ and anti-dsDNA antibodies is reduced by NCS 613 treatment. Prolong PDE4 inhibition in MRL/lpr treated mice is accompanied by ameliorations of kidney damage. Anti-dsDNA antibody-induced mesangial cell proliferation and nephromegaly is prevented by increased kidney cAMP levels (Figure 1). In other study, it has been shown that PDE4 inhibitors improve kidney damage by increasing cAMP levels after renal ischemia-reperfusion injury in rats $^{32}$. Our recent study has shown that PDE4 inhibition by NCS 613 exerts antiproliferative effects on A549 lung epithelial cells and human lung adenocarcinoma explants, as well as anti-proliferative effects on mesangial cells ${ }^{29,33}$. From animal studies to patient bed site, our translational observations strongly support the idea that inhibition of

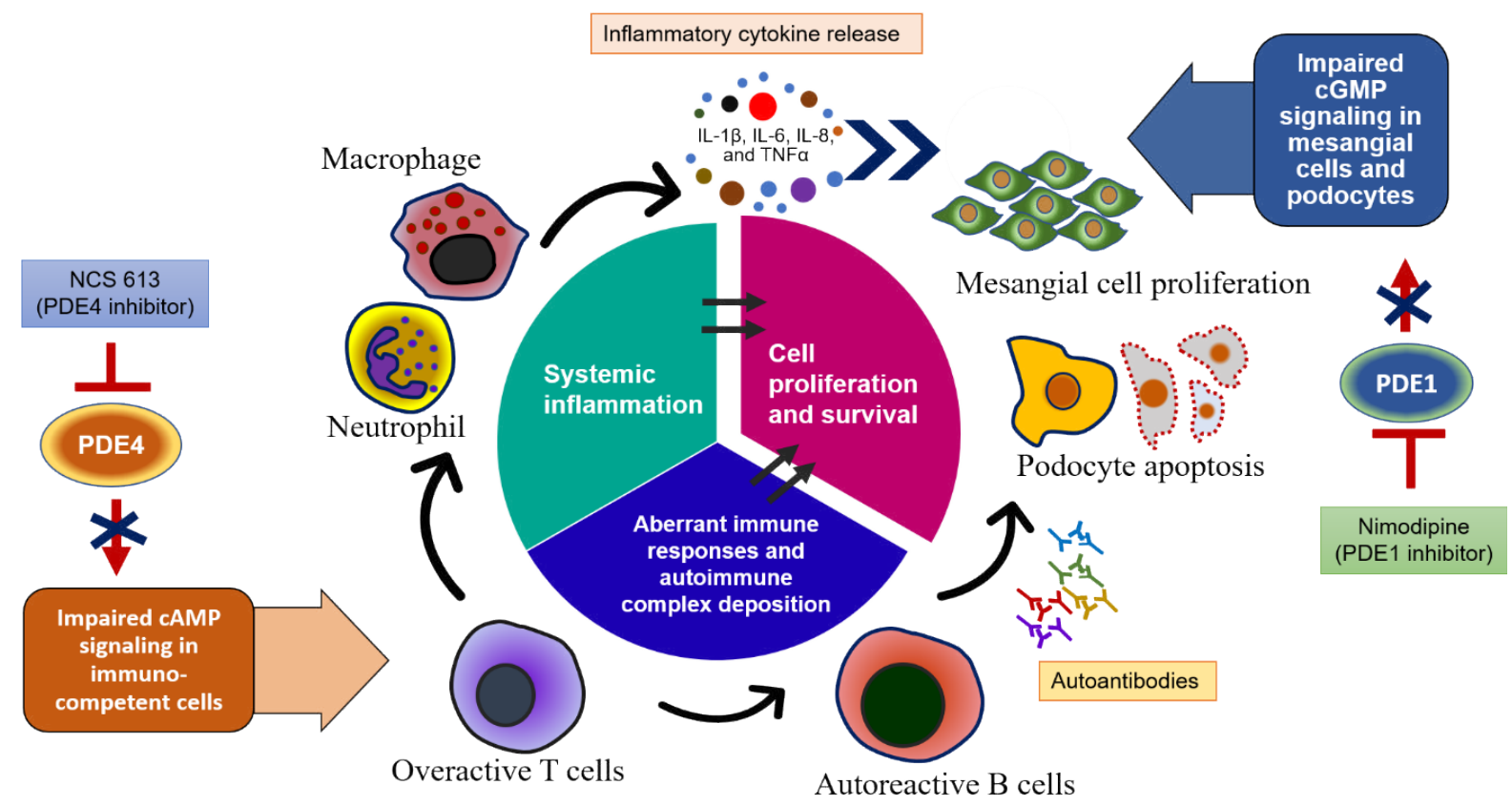

Figure 1: Contributions of cyclic nucleotide signaling pathways to lupus nephritis pathogenesis. Lower cAMP levels in immunocompetent cells induced T cells overactivity, storm of inflammatory cytokine release by inflammatory cells, and autoantibody production by $B$ cells. Immune complexes and inflammatory cytokines mediate mesangial cell proliferation and podocyte apoptosis leading to kidney dysfunction. PDE4 inhibition by NCS 613 prevents proinflammatory signaling whereas PDE1 inhibition induces anti-lymphoproliferative effects. Dual inhibition of PDE1 and PDE4 may represent a promising therapeutic approach to tackle LN. 
PDE4 activities in peripheral mononuclear cells (PBMCs) can control proinflammatory signaling. The PBMCs consist of monocytes and lymphocytes that play key roles in SLE systemic inflammatory processes. LPS stimulations predominantly activate TRL4 signaling in macrophages and release of large amounts of TNF $\alpha$. Inhibition of PDE4B and PDE4C by NCS 613 reduces p38 MAPK and NFkB signaling and ultimately prevents IL-6, IL-8, and TNF $\alpha$ secretion upon PBMCs stimulation by LPS. By controlling systemic inflammation mediated by macrophages and production of autoantibodies by B cells, NCS 613 treatment has been shown to be an attractive therapeutic intervention in management of SLE.

\section{PDE1, the Major cGMP Degrading Enzyme in Lupus Nephritis}

Mesangial cell proliferation plays an important role in the pathogenesis of progressive glomerular abnormalities, leading to glomerulosclerosis and kidney dysfunctions ${ }^{8}$. PDE1 sub-family of enzymes hydrolyze both cAMP and cGMP in a mutually competitive manner, ${ }^{9}$. PDE1 involves in cell proliferation by activating p27Kip1 and regulating cell cycle ${ }^{35,36}$. We previously documented splenomegaly, nephromegaly, and hepatomegaly in MRL/lpr mice. This correlates with an increase PDE1 activity in the kidney and liver, and PDE2 activity in the spleen. Calcium/ calmodulin activated-PDE1 has been reported to play an important role in cell proliferation and is implicated in rodent heart hypertrophy ${ }^{37}$. Inhibition of PDE1A isoform results in vascular remodeling and inhibition of smooth muscle cells (SMCs) proliferation in lung vasculature ${ }^{36}$. Other reports have discussed the anti-proliferative and anti-cancer effects of PDE1 inhibitors that will not be discussed in this manuscript ${ }^{35,38}$. From 8 week to week 15 of age MRL/lpr lupus-prone mice develop nephromegaly. In healthy mice, PDE1 is the major cGMP hydrolyzing enzyme in the kidney contributing to $52 \%$ of the total cGMP-PDE activities while the rest is due to PDE2, PDE3, and PDE5. The expression of three PDE1 isoforms (PDE1A, PDE1B, and PDE1C) is critical to cGMP breakdown in the kidney. During lupus disease progression in lupus prone mice kidney, cGMP-PDE activities significantly increase in the kidney, mainly due to PDE1 activity which lowers intracellular cGMP levels. Of critical importance PDE1B and PDE1C upregulation seems to play critical role in kidney pathology. Expression of PDE1 particularly increases in the glomerulus supporting the idea that this isoform might contribute to mesangial cell proliferation. Implications of cGMP-PDE in lymphoproliferative phenotype and organomegaly were tested with the used of PDE1and PDE2 inhibitors. Intravenous administration of Nimodipine (PDE1 inhibitor) to MRL/lpr mice but not of EHNA (PDE2 inhibitor) was able to significantly lower peripheral leucocyte hypercellularity, a characteristic feature of lupus- prone mice. PDE1 inhibition is a potential therapeutic target for prevention of abnormal renal remodeling leading to kidney dysfunction. Future investigation should address how the simultaneous inhibition of PDE4 and PDE1 could ameliorate nephritis complications in SLE.

\section{Conclusion and Perspective}

PDE1 and PDE4 predominantly degrade kidney cGMP and cAMP respectively. Cyclic nucleotide signaling pathways are impaired in lupus nephritis due to PDE1 and PDE4 upregulation. PDE4 activity contribute tolower kidneycAMP and inflammation, while PDE1 promotes lower cGMP and mesangial cell proliferation. The pathogenic autoimmune complexes trigger immune response, podocyte apoptosis, and mesangial cell proliferation thereby exacerbating kidney dysfunction. Dual inhibition of PDE1 and PDE4 may represent a unique therapeutic approach to tackle systemic inflammation, anti-proliferative effects on mesangial cells, and kidney hypertrophy, while improving disease outcome.

\section{Conflict of Interest}

The author declared no conflict of interest.

\section{References}

1. Monneaux F, and Muller S. Molecular therapies for systemic lupus erythematosus: clinical trials and future prospects. Arthritis Res Ther. 2009; 11(3): 234.

2. Obrisca B, Sorohan B, Tuta L, et al. Advances in Lupus Nephritis Pathogenesis: From Bench to Bedside. Int J Mol Sci. 2021; 22(7).

3. Bhargava R, Lehoux S, Maeda $\mathrm{K}$, et al. Aberrantly glycosylated IgG elicits pathogenic signaling in podocytes and signifies lupus nephritis. JCI Insight. 2021; 6(9).

4. Jin S, Yu C, and Yu B. Changes of serum IL-6, IL-10 and TNF-alpha levels in patients with systemic lupus erythematosus and their clinical value. Am J Transl Res. 2021; 13(4): 2867-74.

5. Yougbare I, Keravis T, Abusnina A, et al. Cyclic GMP catabolism upregulation in MRL/lpr lupus-prone mice is associated with organ remodeling. Biochim Biophys Acta. 2014; 1842(7): 916-26.

6. Arazi A, Rao DA, Berthier CC, et al. The immune cell landscape in kidneys of patients with lupus nephritis. Nat Immunol. 2019; 20(7): 902-14.

7. Sakkas LI, Mavropoulos A, and Bogdanos DP. Phosphodiesterase 4 Inhibitors in Immune-mediated Diseases: Mode of Action, Clinical Applications, Current and Future Perspectives. Curr Med Chem. 2017; 24(28): 3054-67.

8. Cheng J, Thompson MA, Walker HJ, et al. Differential regulation of mesangial cell mitogenesis by cAMP phosphodiesterase isozymes 3 and 4. Am J Physiol Renal Physiol. 2004; 287(5): F940-53.

9. Samidurai A, Xi L, Das A, et al. Role of phosphodiesterase 1 in the pathophysiology of diseases and potential therapeutic opportunities. Pharmacol Ther. 2021; 226: 107858.

10. Gorjestani S, Rider V, Kimler BF, et al. Extracellular signal-regulated kinase $1 / 2$ signalling in SLE T cells is influenced by oestrogen and disease activity. Lupus. 2008; 17(6): 548-54.

11. Yougbare I, Boire G, Roy M, et al. NCS 613 exhibits anti-inflammatory effects on PBMCs from lupus patients by inhibiting p38 MAPK and NF-kappaB signalling pathways while reducing proinflammatory cytokine production. Can J Physiol Pharmacol. 2013; 91(5): 353-61. 
12. Abdelnabi HH. Is podocytopathy another image of renal affection in p-SLE? Pediatr Rheumatol Online J. 2021; 19(1): 57.

13. Wang SF, Chen YH, Chen DQ et al. Mesangial proliferative lupus nephritis with podocytopathy: a special entity of lupus nephritis. Lupus. 2018; $27(2)$ : 303-11.

14. Tahseldar-Roumieh R, Ghali-Ghoul R, Lugnier C, et al. Effect of phosphodiesterase 5 inhibitor on alteration in vascular smooth muscle sensitivity and renal function in rats with liver cirrhosis. $A m J$ Physiol Heart Circ Physiol. 2006; 290(1): H481-8.

15. Lugnier C. Cyclic nucleotide phosphodiesterase (PDE) superfamily: a new target for the development of specific therapeutic agents. Pharmacol Ther. 2006; 109(3): 366-98.

16. Shen K, Johnson DW, and Gobe GC. The role of cGMP and its signaling pathways in kidney disease. Am J Physiol Renal Physiol. 2016; 311(4): F671-F81.

17. Filipak M, Sparks RL, Tzen CY, et al. Tumor necrosis factor inhibits the terminal event in mesenchymal stem cell differentiation.J Cell Physiol. 1988; $137(2): 367-73$

18. Houslay MD, Baillie GS, and Maurice DH. cAMP-Specific phosphodiesterase-4 enzymes in the cardiovascular system: a molecular toolbox for generating compartmentalized cAMP signaling. Circ Res. 2007; 100(7): 950-66.

19. Paes D, Schepers M, Rombaut B, et al. The Molecular Biology of Phosphodiesterase 4 Enzymes as Pharmacological Targets: An Interplay of Isoforms, Conformational States, and Inhibitors. Pharmacol Rev. 2021; 73(3): 1016-49.

20. Tahseldar-Roumieh R, Keravis T, Maarouf S, et al. PDEs1-5 activity and expression in tissues of cirrhotic rats reveal a role for aortic PDE3 in NO desensitization. Int J Exp Pathol. 2009; 90(6): 605-14.

21. Schinner E, Wetzl V, and Schlossmann J. Cyclic nucleotide signalling in kidney fibrosis. Int J Mol Sci. 2015; 16(2): 2320-51.

22. Xing Q Su H, Cui J, et al. Role of Treg cells and TGF-beta1 in patients with systemic lupus erythematosus: a possible relation with lupus nephritis. Immunol Invest. 2012; 41(1): 15-27.

23. Theofilopoulos AN, and Dixon FJ. Murine models of systemic lupus erythematosus. Adv Immunol. 1985; 37: 269-390.

24. Cohen PL, and Eisenberg RA. Lpr and gld: single gene models of systemic autoimmunity and lymphoproliferative disease. Annu Rev Immunol. 1991; 9: 243-69.

25. Jin SL, and Conti M. Induction of the cyclic nucleotide phosphodiesterase PDE4B is essential for LPS-activated TNFalpha responses. Proc Natl Acad Sci U S A. 2002; 99(11): 762833.
26. Lugnier C, Meyer A, Talha S, et al. Cyclic nucleotide phosphodiesterases: New targets in the metabolic syndrome? Pharmacol Ther. 2020; 208: 107475.

27. Boichot E, Wallace JL, Germain N, et al. Anti-inflammatory activities of a new series of selective phosphodiesterase 4 inhibitors derived from 9-benzyladenine. J Pharmacol Exp Ther. 2000; 292(2): 647-53.

28. Yougbare I, Morin C, Senouvo FY, et al. NCS 613, a potent and specific PDE4 inhibitor, displays anti-inflammatory effects on human lung tissues. Am J Physiol Lung Cell Mol Physiol. 2011; 301(4): L441-50.

29. Yougbare I, Belemnaba L, Morin C, et al. NCS 613, a Potent PDE4 Inhibitor, Displays Anti-Inflammatory and Anti-Proliferative Properties on A549 Lung Epithelial Cells and Human Lung Adenocarcinoma Explants. Front Pharmacol. 2020; 11: 1266.

30. Yougbare I, McTague A, He L, et al. Anti-FIM and Anti-FHA Antibodies Inhibit Bordetella pertussis Growth and Reduce Epithelial Cell Inflammation Through Bacterial Aggregation. Front Immunol. 2020; 11: 605273 .

31. De Souza A, Strober BE, Merola JF, et al. Apremilast for discoid lupus erythematosus: results of a phase 2, open-label, single-arm, pilot study. J Drugs Dermatol. 2012; 11(10): 1224-6.

32. Okusa MD, Linden J, Huang L, et al. Enhanced protection from renal ischemia-reperfusion [correction of ischemia:reperfusion] injury with $\mathrm{A}(2 \mathrm{~A})$-adenosine receptor activation and PDE 4 inhibition. Kidney Int. 2001; 59(6): 2114-25.

33. Lugnier C, Al-Kuraishy HM, and Rousseau E. PDE4 inhibition as a therapeutic strategy for improvement of pulmonary dysfunctions in Covid-19 and cigarette smoking. Biochem Pharmacol. 2021; 185: 114431.

34. Lugnier C. PDE inhibitors: a new approach to treat metabolic syndrome? Curr Opin Pharmacol. 2011; 11(6): 698-706.

35. Abusnina A, Alhosin M, Keravis T, et al. Down-regulation of cyclic nucleotide phosphodiesterase PDE1A is the key event of p73 and UHRF1 deregulation in thymoquinone-induced acute lymphoblastic leukemia cell apoptosis. Cell Signal. 2011; 23(1): 152-60.

36. Miller CL, Cai Y, Oikawa M, et al. Cyclic nucleotide phosphodiesterase 1A: a key regulator of cardiac fibroblast activation and extracellular matrix remodeling in the heart. Basic Res Cardiol. 2011; 106(6): 102339.

37. Miller CL, Oikawa M, Cai Y, et al. Role of Ca2+/calmodulin-stimulated cyclic nucleotide phosphodiesterase 1 in mediating cardiomyocyte hypertrophy. Circ Res. 2009; 105(10): 956-64.

38. Abusnina A, Keravis T, Yougbare I, et al. Anti-proliferative effect of curcumin on melanoma cells is mediated by PDE1A inhibition that regulates the epigenetic integrator UHRF1. Mol Nutr Food Res. 2011; 55(11): 1677-89. 